\title{
X-Ray Variability of NLS1s and BLS1s
}

\author{
Rick Edelson ${ }^{\text {a }}$ \\ ${ }^{a}$ UCLA Department of Physics \&5 Astronomy; Los Angeles, CA 90095-1562; USA
}

\begin{abstract}
Evenly-sampled hard X-ray monitoring was obtained with RXTE for one NLS1 (Akn 564) and four BLS1s. The variability amplitude of the NLS1 was no larger than the mean of the BLS1s, and the NLS1 showed stronger variability in the harder portion of the RXTE band, while the BLS1s were more strongly variable in the relatively soft part of the band. This contribution discusses possible explanations for these surprising results, including possible calibration errors or systematic differences between long and short time scale variability in NLS1s and BLS1s.
\end{abstract}

Key words: galaxies: active; quasars: general; quasars: absorption lines; X-rays: galaxies

\section{Introduction}

Narrow-Line Seyfert 1s (NLS1s; see, e.g., Boller, Brandt \& Fink 1996) show much steeper and variable soft X-ray emission and narrower optical permitted lines than Broad-Line Seyfert 1s (BLS1s). This is in many ways analagous to the behavior of Galactic Black Hole Candidates (GBHCs) in the sense that GBHCs in their high state are similar to NLS1s while those in the low state are similar to BLS1s (e.g., Pounds et al. 1995). As it has been argued that the high-state GBHCs represent accretion near or above the Eddington rate, this may suggest that the extreme properties of NLS1s also result from high accretion rates (e.g., Vaughan et al. 1999).

This analogy is good regarding the spectral similarities between Seyfert 1s and NLS1s, but it is generally not appreciated that (at least based on our relatively poorly defined current knowledge base), it breaks down when variability is considered. It appears that the soft X-ray variability of NLS1s is stronger than that of BLS1s (e.g., Turner et al. 1999), but for GBHCs, it is the low-state objects that have the stronger variability. (In fact, there is also the behavior 
in the very high state (Van Paradijs 1998), but this is not directly addressed in this analogy.)

In this study, the initial results from a systematic hard X-ray variability survey undertaken with the Rossi X-ray Timing Explorer (RXTE) were studied. This work focused on BLS1s but did include one NLS1 (Akn 564), as the steepspectrum NLS1s are much weaker and less numerous than BLS1s in the hard X-rays. As discussed below, the NLS1 showed behavior that was not what was naively expected on the basis of the soft X-ray results. However, we emphasize that this is a very preliminary result based on a single NLS1, so no firm conclusions can yet be drawn.

\section{Data}

As part of a program to study the fluctuation power density (PDS) of Seyfert 1 galaxies, even sampling (every $4.3 \mathrm{~d}$ ) was obtained for five BLS1s (NGC 3516, NGC 3783, NGC 4151, NGC 5548 and Fairall 9) and 1 NLS1 (Akn 564). As of this writing, the first 9 months of data have been processed by the RXTE Science Operations Center and used in this contribution.

\section{Light Curves and Excess Variance Analysis}

These data were analyzed to produce $16 \mathrm{~s}$ light curves by standard techniques, as discussed, e.g., in Edelson et al. (2000). These data were then binned up by orbit to produce the light curves shown in Figure 1. The excess variance (e.g., Turner et al. 1999) has been computed for each of these data sets (gathered with near-identical sampling) and listed to the right of each panel. Note that the excess variance of the one NLS1, Akn 564, is by no means extreme, but is instead near the middle of the distribution of the BLS1s.

One might have expected the NLS1 to have a much larger excess variance, as indeed has been reported in the short time scale, softer ROSAT and ASCA data (e.g., Turner et al. 1999). There are a number of possible explanations of this, the most obvious being the strong caution that this has only been seen in a single object. In spite of the difficulties in making such a study in the hard X-rays, as discussed below, it is expected that similarly-sampled RXTE observations of a second NLS1, Ton S180, should be available soon.

Two additional differences with the ASCA (or ROSAT) data could have contributed to this as well: First, those satellites sampled the softer X-rays, and it is in the soft X-rays that NLS1s and BLS1s show their greatest spectral 


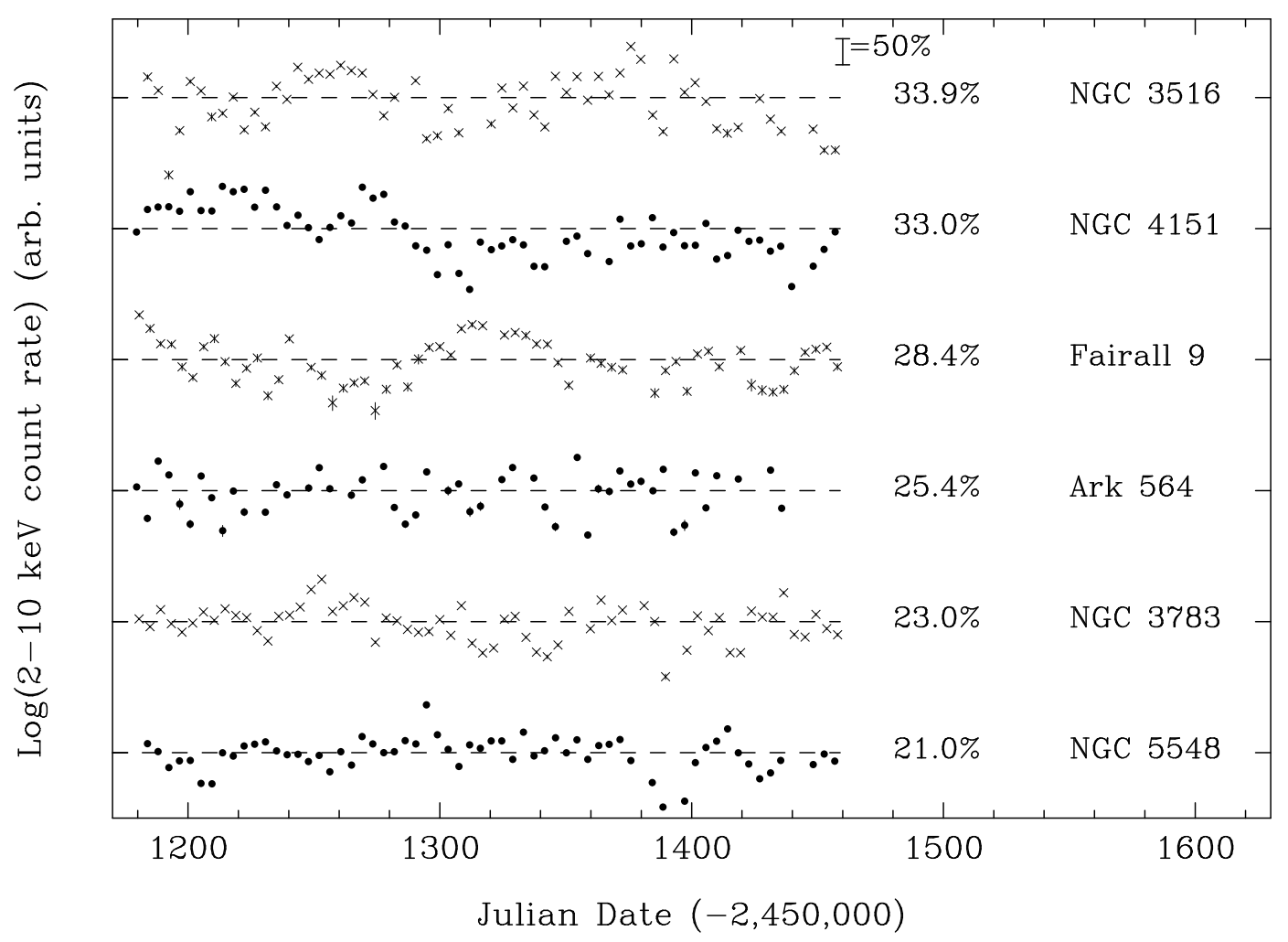

Fig. 1. Light curves for the six target Seyfert galaxies. Note that the one NLS1, Akn 564 , does not show unusually strong hard X-ray variability.

slope divergance as well. Second, the fact that RXTE was designed and executed with much more relaxed and cooperative scheduling constraints makes it more well-suited to study the longer time scales. It is possible that processes that dominate the short time scales could be different than those that are important over longer time scales.

\section{Spectral Variability}

A second unexpected but tentative result is shown in Figure 2, which plots the relatively softer RXTE band (2-4 keV) against the harder 7-10 keV band. (The 4-7 keV band was avoided to minimize the contribution from any iron line.) All of the BLS1s show stronger variability at softer energies, while the NLS1 shows the opposite: stronger variability in the harder bands. This is not consistent with the idea that the soft X-rays are more strongly variable for NLS1s, but there is a possible instrumental problem because the NLS1 Akn $564($ about $1 \mathrm{c} / \mathrm{s}$ ) is much fainter than the BLS1s . Unfortunately, there are some serious problems with the background models as a function of energy, which is important because RXTE is a non-imaging instrument. All of the BLS1s show stronger variability at softer energies, while the NLS1 shows the 
opposite: stronger variability in the harder bands. Sufficient gaps exist in the current understanding of the RXTE background model to make it impossible to rule out systematic errors as the cause of this effect.
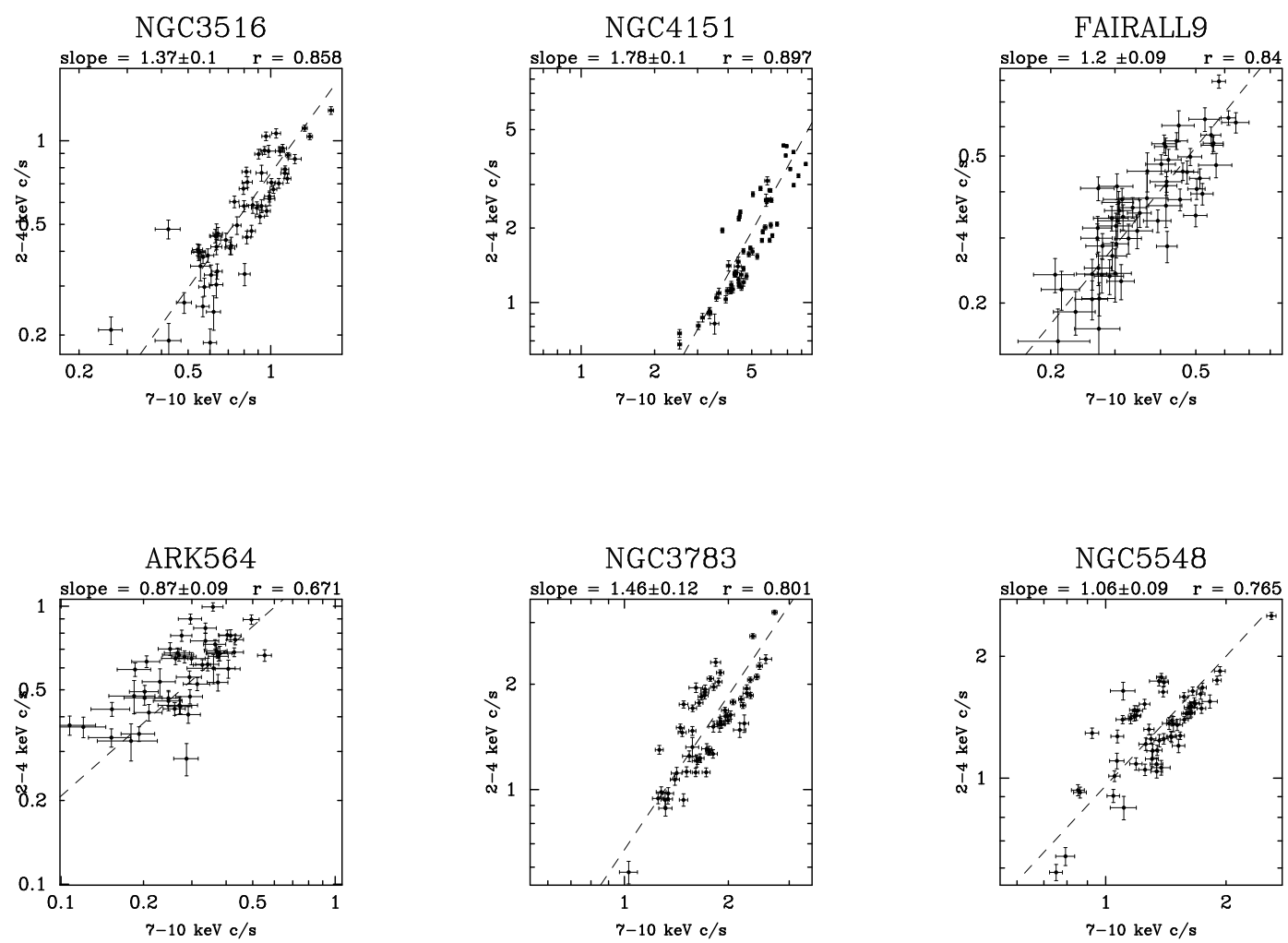

Fig. 2. Plots of hard and soft band count rates for the six target Seyfert galaxies. Note that the one NLS1 shows stronger variability in the hardest band.

\section{References}

[1] Boller, T., Brandt, N. \& Fink, H. 1996, A\&A, 305, 5

[2] Edelson, R. et al. 2000, ApJ, in press, astro-ph/9912266

[3] Pounds, K. et al. 1995, MNRAS, 277, L5

[4] Turner, T. J. et al. 1999, ApJ, 510, 178

[5] Van Paradijs, J. 1998, The Many Faces of Neutron Stars, eds. R. Buccheri, J. van Paradijs, \& M. A. Alpar (Boston:Kluwer), p. 279

[6] Vaughan, S. et al. 1999, MNRAS, 309, 113 